\title{
ARTINIAN AND NOETHERIAN PARTIAL SKEW GROUPOID RINGS
}

\author{
PATRIK NYSTEDT \\ University West, Department of Engineering Science, SE-46186 Trollhättan, Sweden \\ JOHAN ÖINERT \\ Blekinge Institute of Technology, Department of Mathematics and Natural Sciences, \\ SE-37179 Karlskrona, Sweden \\ HÉCTOR PINEDO \\ Universidad Industrial de Santander, Escuela de Matemáticas, Carrera 27 Calle 9, \\ Edificio Camilo Torres Apartado de correos 678, Bucaramanga, Colombia
}

\begin{abstract}
Let $\alpha=\left\{\alpha_{g}: R_{g^{-1}} \rightarrow R_{g}\right\}_{g \in \operatorname{mor}(G)}$ be a partial action of a groupoid $G$ on a non-associative ring $R$ and let $S=R \star_{\alpha} G$ be the associated partial skew groupoid ring. We show that if $\alpha$ is global and unital, then $S$ is left (right) artinian if and only if $R$ is left (right) artinian and $R_{g}=\{0\}$, for all but finitely many $g \in \operatorname{mor}(G)$. We use this result to prove that if $\alpha$ is unital and $R$ is alternative, then $S$ is left (right) artinian if and only if $R$ is left (right) artinian and $R_{g}=\{0\}$, for all but finitely many $g \in \operatorname{mor}(G)$. Both of these results apply to partial skew group rings, and in particular they generalize a result by J. K. Park for classical skew group rings, i.e. the case when $R$ is unital and associative, and $G$ is a group which acts globally on $R$. Moreover, we provide two applications of our main result. Firstly, we generalize I. G. Connell's classical result for group rings by giving a characterization of artinian (non-associative) groupoid rings. This result is in turn applied to partial group algebras. Secondly, we give a characterization of artinian Leavitt path algebras. At the end of the article, we use globalization to analyse noetherianity and artinianity of partial skew groupoid rings as well as establishing two Maschke-type results, thereby generalizing results by Ferrero and Lazzarin from the group graded case to the groupoid situation.
\end{abstract}

E-mail addresses: patrik.nystedt@hv.se; johan.oinert@bth.se; hpinedot@uis.edu.co.

Date: October 12, 2016.

2010 Mathematics Subject Classification. 16S35, 16S99, 16P20, 16N99, 17A05, 17 A99.

Key words and phrases. artinian ring, noetherian ring, partial skew groupoid ring, partial skew group ring, partial group algebra, Leavitt path algebra, globalization, Morita equivalence. 


\section{INTRODUCTION}

In 1963, I. G. Connell [8] showed that if $R$ is an associative and unital ring, and $G$ is a group, then the group ring $R[G]$ is left (right) artinian if and only if $R$ is left (right) artinian and $G$ is finite. Later on, D. S. Passman gave examples of artinian twisted group rings by infinite groups (see [36, Section 4]). Passman's examples show that Connell's result can not be generalized to twisted group rings or, more generally, crossed products.

Another type of crossed products, generalizing group rings, are the skew group rings. Recall that if $\alpha: G \ni g \mapsto \alpha_{g} \in \operatorname{Aut}(R)$ is a group homomorphism from $G$ to $\operatorname{Aut}(R)$, the group of ring automorphisms of $R$, then the skew group ring $R *_{\alpha} G$ is the set of finite

formal sums of the form $\sum_{g \in G} r_{g} g$ with addition defined componentwise and multiplication defined by the relations $(r g)(s h)=\left(r \alpha_{g}(s)\right) g h$, for $r, s \in R$ and $g, h \in G$. In 1979, J. K. Park [35] generalized Connell's result to skew group rings by showing the following.

Theorem 1.1 (Park [35]). If $R$ is a unital and associative ring, and $\alpha$ is a group homomorphism from a group $G$ to $\operatorname{Aut}(R)$, then the skew group ring $R *_{\alpha} G$ is left (right) artinian if and only if $R$ is left (right) artinian and $G$ is finite.

In this article, we consider two generalizations (see Theorem 1.2 and Theorem 1.3) of Theorem 1.1 in the context of partial skew groupoid rings over non-associative rings, i.e. rings which are not necessarily associative. Previously, partial skew groupoid rings have been defined only over associative rings. However, since there are many interesting examples of non-associative rings with various types of actions, it is only natural to seek such a theory in this more general sense. For instance, our Theorem 1.2 holds when $R$ equals any of the algebras in the infinite chain of classical Cayley-Dickson doublings: the real numbers $\mathbb{R}$, the complex numbers $\mathbb{C}$, Hamilton's quaternions $\mathbb{H}$, Graves' octonions $\mathbb{O}$, the sedenions $\mathbb{S}$, the trigintaduonions $\mathbb{T}$ etc. Other important classes of examples to which our Theorem 1.2 can be applied comes from the cases when $R$ is a Jordan algebra or a Baric algebra.

The notion of a partial action of a group on a $\mathrm{C}^{*}$-algebra was introduced by R. Exel [13], as an efficient tool to their study. Since then, the theory of (twisted) partial actions on $\mathrm{C}^{*}$-algebras has played a key role in the characterization of several classes of $\mathrm{C}^{*}$-algebras as crossed products by (twisted) partial actions, e.g. AF-algebras [15], Bunce-Deddens algebras [14], Cuntz-Krieger algebras [16] and Cuntz-Li algebras [7], (see also the survey [9]). In a purely algebraic context, partial skew group rings were introduced by M. Dokuchaev and R. Exel [10] as a generalization of classical skew group rings and as an algebraic analogue of partial crossed product $\mathrm{C}^{*}$-algebras.

Partial group actions can be described in terms of premorphisms, which is a notion introduced by McAlister and Reilly [32]. As explained in [25], a partial action of a group $G$ on a set $X$ is a unital premorphism from $G$ to the inverse monoid $\mathcal{I}(X)$, consisting of bijections between subsets of $X$. This perspective motivated to the study of partial actions of other algebraic structures rather than groups on sets, such as semigroups [22, 23, 24, 27, 33, and ordered groupoids [4, 18. Groupoids also appear naturally in the context of partial group actions. Indeed, in [1] the author constructs a functor from the category 
of partial actions to the category of groupoids. In [4, 5], partial actions of groupoids on rings and the corresponding partial skew groupoid rings were introduced, and recently the authors of [21] gave a description of Leavitt Path algebras as partial skew groupoid rings.

Recall that a groupoid $G$ is a small category with the property that all its morphisms are isomorphisms. The family of objects and morphisms of $G$ will be denoted by ob $(G)$ and $\operatorname{mor}(G)$ respectively. As usual one identifies and object $e$ with the identity morphism $\operatorname{Id}_{e}$, so ob $(G) \subseteq \operatorname{mor}(G)$. If $g \in \operatorname{mor}(G)$, then the domain and codomain of $g$ will be denoted by $d(g)$ and $c(g)$, respectively. We let $G^{2}$ denote the set of all pairs $(g, h) \in \operatorname{mor}(G) \times \operatorname{mor}(G)$ that are composable, i.e. such that $d(g)=c(h)$. Let $R$ be a non-associative ring. By this we mean that $R$ is an additive group equipped with a multiplication which is distributive with respect to addition. If $R$ is unital, then the multiplicative identity is denoted by $1_{R}$ and is always assumed to be non-zero. The identity map $R \rightarrow R$ is denoted by $\mathrm{id}_{R}$. $\operatorname{Recall}$ from [5] that $\alpha=\left\{\alpha_{g}: R_{g^{-1}} \rightarrow R_{g}\right\}_{g \in \operatorname{mor}(G)}$ is called a partial action of $G$ on $R$ if for each $g \in \operatorname{mor}(G), R_{g}$ is an ideal of $R_{c(g)}, R_{c(g)}$ is an ideal of $R$ and $\alpha_{g}: R_{g^{-1}} \rightarrow R_{g}$ is a ring isomorphism satisfying the following three axioms:

(P1) if $e \in \mathrm{ob}(G)$, then $\alpha_{e}=\mathrm{id}_{R_{e}}$;

(P2) if $(g, h) \in G^{2}$, then $R_{(g h)^{-1}} \supseteq \alpha_{h}^{-1}\left(R_{h} \cap R_{g^{-1}}\right)$;

(P3) if $(g, h) \in G^{2}$ and $x \in \alpha_{h}^{-1}\left(R_{h} \cap R_{g^{-1}}\right)$, then $\left(\alpha_{g} \circ \alpha_{h}\right)(x)=\alpha_{g h}(x)$.

The associated partial skew groupoid ring $R \star_{\alpha} G$ is the set of all finite formal sums $\sum_{g \in \operatorname{mor}(G)} r_{g} \delta_{g}$, where $r_{g} \in R_{g}$, with addition defined componentwise and multiplication determined by the rule

$$
\left(r_{g} \delta_{g}\right)\left(r_{h}^{\prime} \delta_{h}\right)=\alpha_{g}\left(\alpha_{g^{-1}}\left(r_{g}\right) r_{h}^{\prime}\right) \delta_{g h}
$$

if $(g, h) \in G^{2}$, and $\left(r_{g} \delta_{g}\right)\left(r_{h}^{\prime} \delta_{h}\right)=0$, otherwise. Since the ring structure of $R \star_{\alpha} G$ only depends on the choice of the rings $R_{e}$, for $e \in \mathrm{ob}(G)$, we may take $R$ to be any ring having these rings as ideals. From this point of view, we may therefore assume that the following fourth axiom holds:

(P4) $R=\oplus_{e \in \mathrm{ob}(G)} R_{e}$.

By adding this fourth axiom we get another advantage. Namely, our definition of partial groupoid actions, in the case when $G$ is a group, i.e. when $G$ has one object, now coincides with the classical definition of partial group actions on rings. We say that $\alpha$ is unital if every non-zero $R_{g}$ is unital, for $g \in \operatorname{mor}(G)$. The action $\alpha$ is called global, if $\alpha_{g h}=\alpha_{g} \alpha_{h}$, for $(g, h) \in G^{2}$. It follows from [5, Lemma 1.1(i)] that $\alpha$ is global, if and only if $R_{g}=R_{c(g)}$, for all $g \in \operatorname{mor}(G)$. In that case, apart from the associativity requirement on $R, R \star_{\alpha} G$ coincides with the definition of a skew groupoid ring given in [31]. Here is an outline of the article.

In Section 2, we recall some notions and results from non-associative ring theory that we need in the sequel. In Section 3, we show the following generalizations of Theorem 1.1.

Theorem 1.2. If $\alpha$ is a global unital action of a groupoid $G$ on a non-associative ring $R$, then the partial skew groupoid ring $R \star_{\alpha} G$ is left (right) artinian, if and only if, $R$ is left (right) artinian and $R_{g}=\{0\}$, for all but finitely many $g \in \operatorname{mor}(G)$. 
Theorem 1.3. If $\alpha$ is a unital partial action of a groupoid $G$ on an alternative ring $R$, then the partial skew groupoid ring $R \star_{\alpha} G$ is left (right) artinian, if and only if, $R$ is left (right) artinian and $R_{g}=\{0\}$, for all but finitely many $g \in \operatorname{mor}(G)$.

A couple of remarks are needed here. First, notice that the condition that $R_{g}=\{0\}$ for all but finitely many $g \in \operatorname{mor}(G)$ can not, in general, be replaced by the statement that $\operatorname{mor}(G)$ is finite in Theorem [1.2, nor in Theorem [1.3. To see this, let $G$ be any infinite groupoid and let $K$ be any left (or right) artinian non-associative ring, e.g. a field. Fix $e \in \mathrm{ob}(G)$ and put $R_{e}=K$. If $g \in \operatorname{mor}(G)$ and $g \neq e$, then put $R_{g}=\{0\}$. Let $\alpha_{e}=\mathrm{id}_{K}$ and for $g \in \operatorname{mor}(G)$ with $g \neq e$, then let $\alpha_{g}: R_{g^{-1}} \rightarrow R_{g}$ be the zero map. Then $R \star_{\alpha} G=K$ which is left (or right) artinian even though $\operatorname{mor}(G)$ is infinite.

Secondly, it follows by [5, Theorem 2.1] that any unital partial groupoid $\alpha$ action on a unital ring $R$ admits a globalization $\beta$ on a $\operatorname{ring} T$, provided that $R$ is associative. But according to [17, Example 1.4] one can not guarantee that $T$ is unital, not even in the group case. Therefore, a Morita equivalence can in general not be used to deduce Theorem 1.3 from Theorem 1.2 (see Corollary 6.7).

In the following two sections we give some applications of our main results. Namely, in Section 4 we generalize Connell's result and provide a characterization of left (right) artinian groupoid rings (see Theorem 4.1). We also characterize left (right) artinian generalized matrix rings (see Corollary 4.2) and show an analogue of Connell's result for partial group algebras (Corollary 4.3)

In Section 5 we recall the definition of a Leavitt path algebra and briefly explain how it can be viewed as a partial skew group ring, using [20]. Thereafter, we apply our main results and provide a characterization of artinian (and semisimple) Leavitt path algebras (see Theorem 5.2).

In Section 6, we use globalization and Morita equivalence to deduce necessary and sufficient conditions for the artinianity and noetherianity of partial skew groupoid rings, as well as establishing two Maschke-type results (see Theorem 6.11 and Corollary 6.14).

\section{Preliminaries on Rings and Modules}

In this section, we recall some results from non-associative ring theory that we need in the sequel. Throughout this section, let $R$ and $S$ denote non-associative rings with $R$ a subring of $S$. Recall that the center of $R$, denoted by $Z(R)$, is the set of elements in $R$ that commute and associate with all elements of $R$. In other words, an element $x \in R$ belongs to $Z(R)$ precisely when for every choice of $r, r^{\prime} \in R$, we have that $x r=r x,(x r) r^{\prime}=x\left(r r^{\prime}\right)$, $(r x) r^{\prime}=r\left(x r^{\prime}\right)$ and $\left(r r^{\prime}\right) x=r\left(r^{\prime} x\right)$.

Proposition 2.1. If $R$ is unital and simple, then $Z(R)$ is a field.

Proof. See e.g. [34, Proposition 9].

By a left $R$-module we mean an additive group $M$ equipped with a biadditive map $R \times M \ni(r, m) \mapsto r m \in M$. In that case, if $R$ is unital and $1_{R} m=m$, for $m \in M$, then we say that $M$ is unital as a left $R$-module. By a left $R$-submodule of $M$ we mean an additive subgroup $N$ of $M$ such that if $n \in N$ and $r \in R$, then $r n \in N$. Recall that $M$ is called 
artinian if it satisfies the descending chain condition on its poset of submodules. The ring $R$ is called left artinian if it is artinian as a left module over itself. The concepts of right module and right artinian ring are defined analogously. If the ring $R$ is both left and right artinian, then it is said to be artinian.

Proposition 2.2. Suppose that $M$ is a left (right) $S$-module. If $M$ is artinian as a left (right) $R$-module, then $M$ is artinian as a left (right) $S$-module.

Proof. This follows immediately from the fact that $R \subseteq S$.

Proposition 2.3. Suppose that $R$ is a direct summand of the left (right) $R$-module $S$. Then, for any right (left) ideal $I$ of $R$, the relation $I S \cap R=I R$ ( $S I \cap R=R I$ ) holds. In particular, if $S$ is right/left artinian (noetherian) and $R$ is unital, then $R$ is right/left artinian (noetherian).

Proof. Let $T$ be a left (right) $R$-module such that $S=R \oplus T$ as left (right) $R$-modules. Let $I$ be a right (left) ideal of $R$. Then $I S \cap R=[I(R \oplus T)] \cap R=[I R \oplus I T] \cap R=I R$, $(S I \cap R=R I)$. In particular, if $R$ is unital, then we get $I R=I(R I=I)$ from which the last part follows.

Proposition 2.4. Let $R$ be a unital ring. Suppose that $M$ is a left (right) $R$-module and that $N$ is a left (right) $R$-submodule of $M$. Then $M$ is artinian (noetherian), if and only if, $N$ and $M / N$ are artinian (noetherian).

Proof. See [37, Proposition 0.2.19].

Proposition 2.5. Let $R$ be a unital ring. Suppose that $M$ is a left (right) $R$-module. If $M_{1}, \ldots, M_{n}$ are left (right) $R$-submodules of $M$ such that $M=M_{1} \oplus \cdots \oplus M_{n}$, then $M$ is artinian (noetherian) if and only if for each $i \in\{1, \ldots, n\}, M_{i}$ is artinian (noetherian).

Proof. We can use Proposition 2.4. The "only if" statement is clear. For the "if" statement, consider the case $i=2$ and notice that $M_{1}$ is a left (right) $R$-submodule of $M_{1} \oplus M_{2}$ and that $\left(M_{1} \oplus M_{2}\right) / M_{1} \cong M_{2}$. The general case now follows by induction over $i$.

The term ideal refers to two-sided ideal, unless otherwise stated. The ring $R$ is called simple if $\{0\}$ and $R$ are the only ideals of $R$. An ideal $I$ of $R$ is called maximal if $I \subsetneq R$ and for every ideal $J$ of $R$ with $I \subseteq J \subsetneq R$, the relation $I=J$ holds. Note that an ideal $I$ of $R$ is maximal if and only if $R / I$ is simple. The ring $R$ is called semisimple if it is a finite direct sum of simple rings. Following [8], the intersection of all maximal ideals of $R$, denoted by $\mathcal{S}(R)$, will be called the simplicial radical of $R$.

Proposition 2.6. If $R$ is unital and artinian, then $R / \mathcal{S}(R)$ is semisimple.

Proof. See [12, Theorem 1.7 on p. 6].

Recall that $R$ is called alternative if for all $x, y \in R$, the relations $x^{2} y=x(x y)$ and $x y^{2}=(x y) y$ hold. If $R$ is alternative one may define a radical of $R$, denoted $\mathcal{J}(R)$, similar to the Jacobson radical from the associative situation. In the alternative setting $\mathcal{J}(R)$ is often called the Zhevlakov radical of $R$ (see [39, p. 210]). In that case, $\mathcal{J}$ is hereditary in the following sense. 
Proposition 2.7. If $R$ is artinian and $I$ is an ideal of $R$, then $\mathcal{J}(I)=I \cap \mathcal{J}(R)$.

Proof. See [39, Theorem 3 on p. 204].

Proposition 2.8. If $R$ is a unital alternative left (right) artinian ring, then $\mathcal{S}(R)=\mathcal{J}(R)$ and $R / \mathcal{J}(R)$ is semisimple.

Proof. For the equality $\mathcal{S}(R)=\mathcal{J}(R)$, see [12, Proposition 1.2(ii) on p. 94]. The semisimplicity of $R / \mathcal{J}(R)$ now follows from Proposition 2.6 (or [39, Corollary on p. 250]).

Remark 1. The proofs of Propositions 2.6] 2.8, as they appear in [12] and [39], presuppose that $R$ is an algebra over a field. However, it is clear from those proofs that the algebra structure of $R$ is not needed, and therefore one can suppose that $R$ is only a ring.

\section{Partial Skew Groupoid Rings}

In this section, we show Theorem 1.2 and Theorem 1.3. We assume that $G$ is a groupoid, that $R$ is a non-associative ring and that $\alpha=\left\{\alpha_{g}: R_{g^{-1}} \rightarrow R_{g}\right\}_{g \in \operatorname{mor}(G)}$ is a partial action of $G$ on $R$.

Proposition 3.1. If $R$ is left (right) artinian, and $R_{g}=\{0\}$ for all but finitely many $g \in \operatorname{mor}(G)$, then $R \star_{\alpha} G$ is left (right) artinian.

Proof. Put $S=R \star_{\alpha} G$ and $S_{0}=\oplus_{e \in \mathrm{ob}(G)} R_{e} \delta_{e}$. Since $R$ is an artinian ring we get by Proposition 2.4 that $R_{c(g)}$ is also an artinian ring, the same proposition implies that that $R_{g} \delta_{g}$ is artinian as a left (right) module over $R_{c(g)} \delta_{c(g)}\left(R_{d(g)} \delta_{d(g)}\right)$. Therefore, for each $g \in \operatorname{mor}(G)$, we get, from Proposition 2.2, that $R_{g} \delta_{g}$ is artinian as a left (right) $S_{0}$-module. Then Proposition 2.5 implies that $S$ is artinian as a left (right) $S_{0}$-module. Thus, by Proposition 2.2, the ring $S$ is left (right) artinian.

Definition 3.2. A subgroupoid of $G$ is a subcategory of $G$ that is a groupoid in itself. Define the subgroupoid $G^{\#}$ of $G$ in the following way. The objects of $G^{\#}$ are all $e \in \operatorname{ob}(G)$ with $R_{e}$ non-zero. The morphisms of $G^{\#}$ are all morphisms $g \in \operatorname{mor}(G)$ with $d(g), c(g) \in$ $\mathrm{ob}\left(G^{\#}\right)$. Put $R^{\#}=\oplus_{e \in \mathrm{ob}\left(G^{\#}\right)} R_{e}$ and let $\alpha^{\#}$ denote the restriction of $\alpha$ to $G^{\#}$. Then $R \star_{\alpha} G=R^{\#} \star_{\alpha \#} G^{\#}$.

Proposition 3.3. If $R \star_{\alpha} G$ is left/right artinian (noetherian), then $\mathrm{ob}\left(G^{\#}\right)$ is finite.

Proof. Put $S=R \star_{\alpha} G$. Seeking a contradiction, suppose that there is an infinite set of different elements $\left\{e_{i}\right\}_{i \in \mathbb{N}}$ in ob $(G)$ such that for every $i \in \mathbb{N}$, there is a non-zero $r_{i} \in R_{e_{i}}$.

Suppose that $S$ is left artinian. Define a set of left ideals $\left\{I_{i}\right\}_{i \in \mathbb{N}}$ of $S$ by $I_{i}=\oplus_{g \in G^{i}} R_{g} \delta_{g}$, for $i \in \mathbb{N}$, where $G^{i}=\left\{g \in \operatorname{mor}(G) \mid d(g) \in\left\{e_{i}, e_{i+1}, e_{i+2}, \ldots\right\}\right\}$. For every $i \in \mathbb{N}$ and $r_{i} \in R_{e_{i}}$, we get that $r_{i} \delta_{e_{i}} \in I_{i} \backslash I_{i+1}$. Hence, $\left\{I_{i}\right\}_{i \in \mathbb{N}}$ is a strictly descending chain of left ideals of $S$, which is a contradiction.

Suppose that $S$ is right artinian. Define a set of right ideals $\left\{I_{i}\right\}_{i \in \mathbb{N}}$ of $S$ by $I_{i}=$ $\oplus_{g \in G^{i}} R_{g} \delta_{g}$, for $i \in \mathbb{N}$, where $G^{i}=\left\{g \in \operatorname{mor}(G) \mid c(g) \in\left\{e_{i}, e_{i+1}, e_{i+2}, \ldots\right\}\right\}$. For each $i \in \mathbb{N}$ and $r_{i} \in R_{e_{i}}$, we get that $r_{i} \delta_{e_{i}} \in I_{i} \backslash I_{i+1}$. Hence, $\left\{I_{i}\right\}_{i \in \mathbb{N}}$ is a strictly descending chain of right ideals of $S$, which is a contradiction. 
Suppose that $S$ is left noetherian. Define the set of left ideals $\left\{I_{i}\right\}_{i \in \mathbb{N}}$ of $S$ by $I_{i}=$ $\oplus_{g \in G^{i}} R_{g} \delta_{g}$, for $i \in \mathbb{N}$, where $G^{i}=\left\{g \in \operatorname{mor}(G) \mid d(g) \in\left\{e_{1}, e_{2}, \ldots, e_{i}\right\}\right\}$. For every $i \in \mathbb{N}$, we get that $r_{i} e_{i+1} \in I_{i+1} \backslash I_{i}$. Hence, $\left\{I_{i}\right\}_{i \in \mathbb{N}}$ is a strictly ascending chain of left ideals of $S$, which is a contradiction.

Suppose that $S$ is right noetherian. Define the set of right ideals $\left\{I_{i}\right\}_{i \in \mathbb{N}}$ of $S$ by $I_{i}=$ $\oplus_{g \in G^{i}} R_{g} \delta_{g}$, for $i \in \mathbb{N}$, where $G^{i}=\left\{g \in \operatorname{mor}(G) \mid c(g) \in\left\{e_{1}, e_{2}, \ldots, e_{i}\right\}\right\}$. For every $i \in \mathbb{N}$, we get that $r_{i+1} e_{i+1} \in I_{i+1} \backslash I_{i}$. Hence, $\left\{I_{i}\right\}_{i \in \mathbb{N}}$ is a strictly ascending chain of right ideals of $S$, which is a contradiction.

Proposition 3.4. If $\alpha$ is unital and $R \star_{\alpha} G$ is left/right artinian (noetherian), then $R$ is left/right artinian (noetherian).

Proof. Put $S=R \star_{\alpha} G$ and $S_{0}=\oplus_{e \in \mathrm{ob}(G)} R_{e} \delta_{e}$. By Proposition 3.3, we can write $S_{0}=$ $\oplus_{i=1}^{n} R_{e_{i}} \delta_{e_{i}}$, for $e_{1}, \ldots, e_{n} \in \mathrm{ob}(G)$ such that $R_{e_{1}}, \ldots, R_{e_{n}}$ are all non-zero. Then $S_{0}$ is unital with multiplicative identity given by $\sum_{i=1}^{n} 1_{R_{e_{i}}} \delta_{e_{i}}$. Since $S_{0}$ is a direct summand in $S$ as left/right $S_{0}$-modules, we get, from Proposition 2.3, that $S_{0}$ is left/right artinian (noetherian). The desired conclusion now follows from (P4).

Take $e, f \in \operatorname{ob}(G)$. We let $G(e, f)$ denote the set of morphisms in $G$ with domain $e$ and codomain $f$. Then the set $G(e, e)$ is a group. We denote this group by $G_{e}$. Notice that $\alpha_{e}=\left\{\alpha_{g}: R_{g^{-1}} \rightarrow R_{g}\right\}_{g \in G_{e}}$ is a partial (group) action of $G_{e}$ on $R_{e}$.

Proposition 3.5. The set $\operatorname{mor}(G)$ is finite, if and only if, ob $(G)$ is finite and for each $e \in \mathrm{ob}(G)$, the group $G_{e}$ is finite.

Proof. The "only if" statement is clear. Now we show the "if" statement. Suppose that $\mathrm{ob}(G)$ is finite and that for each $e \in \mathrm{ob}(G)$, the group $G_{e}$ is finite. Since $\operatorname{mor}(G)=$ $\bigcup_{e, f \in \operatorname{ob}(G)} G(e, f)$, one only needs to show that $G(e, f)$ is finite, for all $e, f \in \mathrm{ob}(G)$. Notice that if $G(e, f)$ is non-empty, then we have a bijection $G_{e} \ni g \mapsto h g \in G(e, f)$, where $h$ is a fixed element in $G(e, f)$. Hence, $G(e, f)$ is finite as desired.

Definition 3.6. Recall that a subring $A$ of $R$ is called $G$-invariant, if for every $g \in \operatorname{mor}(G)$, the inclusion $\alpha_{g}\left(A \cap R_{g^{-1}}\right) \subseteq A \cap R_{g}$ holds. In that case, the restriction of $\alpha_{g}$ to $R_{g^{-1}}^{\prime}:=$ $R_{g^{-1}} \cap A$, for $g \in \operatorname{mor}(G)$, gives rise to a partial action of $G$ on $A$.

Proposition 3.7. If $\alpha$ is global and unital such that $R \star_{\alpha} G$ is left (right) artinian and for each $e \in \operatorname{ob}\left(G^{\#}\right), R_{e}$ is simple, then $\operatorname{mor}\left(G^{\#}\right)$ is finite.

Proof. Put $S=R \star_{\alpha} G=R^{\#} \star_{\alpha^{\#}} G^{\#}$ and take $e \in \mathrm{ob}(G)$ with $R_{e}$ non-zero. Put $F=Z\left(R_{e}\right)$. Then $F$ is $G_{e}$-invariant and since $R_{e}$ is simple, we get, by Proposition 2.1, that $F$ is a field. Let $\beta$ be the induced group action of $G_{e}$ on $F$. Put $T=F \star_{\beta} G_{e}$. We claim that $T$ is a direct summand in $S$ as right (left) $T$-modules. Assume, for a moment, that the claim holds. From Lemma 2.5 it follows that $T$ is left (right) artinian. By Theorem 1.1, we get that $G_{e}$ is finite. From Proposition 3.3 and Proposition 3.5, we have that $\operatorname{mor}\left(G^{\#}\right)$ is finite. Now we show the claim. Since $F$ is a field, there is an $F$-subspace $V$ of $R_{e}$ such that $R_{e}=F \oplus V$ as vector spaces over $F$. Put $T^{\prime}=\left(\oplus_{g \in G(e, e)} V \delta_{g}\right) \oplus\left(\oplus_{g \in \operatorname{mor}(G) \backslash G_{e}} R_{c(g)} \delta_{g}\right)$. It is clear that $S=T \oplus T^{\prime}$ as additive groups. What remains to show now is that $T^{\prime}$ is 
a right (left) $T$-module. We will only show the "right" part since the "left" part can be shown in an analogous manner. To this end, take $f \in F$ and $g \in G_{e}$. If $h \in G_{e}$ and $v \in V$, then $\left(v \delta_{h}\right)\left(f \delta_{g}\right)=v \alpha_{h}(f) \delta_{h g} \in \oplus_{g \in G_{e}} V \delta_{g}$, since $F$ is $G_{e}$-invariant and $V$ is a right $F$-vector space. Suppose now that $h \in \operatorname{mor}(G) \backslash G_{e}$ and $r \in R_{c(h)}$. If $d(h) \neq e$, then $\left(r \delta_{h}\right)\left(f \delta_{g}\right)=0 \in T^{\prime}$. If $d(h)=e$, then $c(h) \neq e$ and hence $h g \in \operatorname{mor}(G) \backslash G_{e}$. Thus $\left(r \delta_{h}\right)\left(f \delta_{g}\right) \in \oplus_{g \in \operatorname{mor}(G) \backslash G_{e}} R_{c(g)} \delta_{g} \subseteq T^{\prime}$.

Proposition 3.8. If $\alpha$ is a unital partial action, $R$ is semisimple and $R \star_{\alpha} G$ is left (right) artinian, then $R_{g}=\{0\}$ for all but finitely many $g \in \operatorname{mor}(G)$.

Proof. Write $R=R_{1} \oplus \cdots \oplus R_{n}$ as a direct sum of simple rings. Put $\bar{n}=\{1, \ldots, n\}$. Then, for every $g \in G, R_{g}$ is the direct sum of a non-empty subset of $\left\{R_{i}\right\}_{i \in \bar{n}}$. Define a new category $\bar{G}$ in the following way. As objects we take all $(i, e) \in \bar{n} \times \mathrm{ob}(G)$ such that $R_{i}$ is a direct summand in $R_{e}$. As morphisms we take all formal expressions of the form ${ }_{j} g_{i}$, for $i, j \in \bar{n}$ and $g \in \operatorname{mor}(G)$, such that $R_{i}$ is a direct summand in $R_{g^{-1}}, R_{j}$ is a direct summand in $R_{g}$ and $\alpha_{g}\left(R_{i}\right)=R_{j}$. Define the domain $d$ and codomain $c$ by the relations $d\left({ }_{j} g_{i}\right)=(i, d(g))$ and $c\left({ }_{j} g_{i}\right)=(j, c(g))$, for ${ }_{j} g_{i} \in \operatorname{mor}(\bar{G})$. The composition of ${ }_{k} g_{j}$ and ${ }_{j} h_{i}$ in $\operatorname{mor}(\bar{G})$ is defined by the relation $\left({ }_{k} g_{j}\right)\left({ }_{j} h_{i}\right)={ }_{k}(g h)_{i}$. Note that since $\alpha_{e}=\mathrm{id}_{R_{e}}$, for each $(i, e) \in \operatorname{ob}(\bar{G}),{ }_{i} e_{i}$ is the identity morphism at $(i, e)$. It is clear that $\bar{G}$ is a groupoid, where for ${ }_{j} g_{i} \in \operatorname{mor}(\bar{G})$, one has $\left({ }_{j} g_{i}\right)^{-1}={ }_{i}\left(g^{-1}\right)_{j}$. For each ${ }_{j} g_{i} \in \operatorname{mor}(\bar{G})$, put $R_{j} g_{i}=R_{j}=R_{j} c(g)_{j}$ and $\bar{\alpha}_{j g_{i}}=p_{j} \circ \alpha_{g} \circ q_{i}$, where $p_{j}: R \rightarrow R_{j}$ is the projection onto the $j$ th coordinate and $q_{i}: R_{i} \rightarrow R$ is the injection defined by inclusion. Then $\bar{\alpha}=\left\{\bar{\alpha}_{j g_{i}}: R_{i g_{j}^{-1}} \rightarrow R_{j g_{i}}\right\}$ is a global action of $G$ on $R$ such that $R \star_{\bar{\alpha}} \bar{G}=R \star_{\alpha} G$. Note that $\bar{G}^{\#}=\bar{G}$. Thus, by Proposition 3.7. we get that $\operatorname{mor}(\bar{G})$ is a finite set. Put $\mathcal{G}=\left\{g \in \operatorname{mor}(G) \mid R_{g}\right.$ is non-zero $\}$. For each $g \in \mathcal{G}$, fix $i(g), j(g) \in \bar{n}$ such that ${ }_{j(g)} g_{i(g)} \in \bar{G}$. It is clear that the map $\mathcal{G} \ni g \mapsto{ }_{j(g)} g_{i(g)} \in \bar{G}$ is injective. Thus, $\mathcal{G}$ is also finite.

Proof of Theorem 1.2. The "if" statement follows from Proposition 3.1. Now we show the only "if" statement. Suppose that $\alpha$ is a global and unital action of a groupoid $G$ on $R$ such that $R \star_{\alpha} G$ is left (right) artinian. We wish to show that $R$ is left (right) artinian and that $R_{g}=\{0\}$ for all but finitely many $g \in \operatorname{mor}(G)$. The first claim follows from Proposition 3.4. Now we show the second claim. For a $\operatorname{ring} S$, put $\bar{S}=S / A(S)$. For a ring isomorphism $f: S \rightarrow T$, let $\bar{f}: \bar{S} \rightarrow \bar{T}$ be the natural map. Since $\alpha$ is a global action of $G$ on $R$, the collection of maps $\bar{\alpha}:=\left\{\bar{\alpha}_{g}: \bar{R}_{g^{-1}} \rightarrow \bar{R}_{g}\right\}_{g \in \operatorname{mor}(G)}$ is a global action of $G$ on $\bar{R}=\oplus_{e \in \mathrm{ob}(G)} \bar{R}_{e}$. Since, for every $g \in \operatorname{mor}(G)$ with $R_{g} \neq\{0\}, R_{g}$ is unital, the ring $\bar{R}_{g}$ is also non-zero and, by Proposition 2.6, semisimple. Since $\bar{R} \star_{\bar{\alpha}} G$ is an epimorphic image of $R \star_{\alpha} G$, we get that $\bar{R} \star_{\bar{\alpha}} G$ is artinian. Now, by Proposition 3.8 we get that $R_{g}=\mathcal{S}\left(R_{g}\right)$ for all but finitely many $g \in \operatorname{mor}(G)$, and the claim follows from the fact that the ideals $R_{g}$ are unital.

Proof of Theorem 1.3. The "if" statement follows from Proposition 3.1. Now we show the "only if" statement. Suppose that $\alpha$ is a partial action of a groupoid $G$ on an alternative ring $R$ such that $R \star_{\alpha} G$ is left (right) artinian. We shall prove that $R$ is left (right) artinian 
and that $R_{g}=\{0\}$, for all but finitely many $g \in \operatorname{mor}(G)$. The first claim follows from Proposition 3.4. Now we show the second claim. For a ring $S$, put $\bar{S}=S / \mathcal{J}(S)$. For a ring isomorphism $f: S \rightarrow T$, let $\bar{f}: \bar{S} \rightarrow \bar{T}$ be the natural map. Take $g \in \operatorname{mor}(G)$. Then $\bar{R}_{g}$ can be viewed as an ideal of $\bar{R}_{c(g)}$. Indeed, the inclusion $i: R_{g} \rightarrow R_{c(g)}$ induces a well defined ring homomorphism $i^{\prime}: R_{g} \rightarrow \bar{R}_{c(g)}$. By Proposition 2.7, we get that $i^{\prime}$ induces a well defined injective ring homomorphism $i^{\prime \prime}: \bar{R}_{g} \rightarrow \bar{R}_{c(g)}$. If we use $i^{\prime \prime}$ to identify each element of $\bar{R}_{g}$ with its image in $\bar{R}_{c(g)}$, then $\bar{R}_{g}$ can be viewed as an ideal of $\bar{R}_{c(g)}$. It is clear that axioms (i)-(v) hold for the collection of maps $\bar{\alpha}:=\left\{\bar{\alpha}_{g}: \bar{R}_{g^{-1}} \rightarrow \bar{R}_{g}\right\}_{g \in \operatorname{mor}(G)}$. Since, for every $g \in \operatorname{mor}(G)$ with $R_{g} \neq\{0\}, R_{g}$ is unital, the ring $\bar{R}_{g}$ is also non-zero and, by Proposition 2.8 , semisimple. Since $\bar{R} \star_{\bar{\alpha}} G$ is an epimorphic image of $R \star_{\alpha} G$, we get that $\bar{R} \star_{\bar{\alpha}} G$ is artinian. The claim now follows from Proposition 3.8 and the fact that $\alpha$ is unital.

\section{Applications to Groupoid rings, Partial group Rings and Matrix Rings}

Let $G$ be a groupoid. Define an equivalence relation $\sim$ on $\mathrm{ob}(G)$ by saying that if $e, f \in \mathrm{ob}(G)$, then $e \sim f$ if there is $g \in \operatorname{mor}(G)$ with $d(g)=e$ and $c(g)=f$. Let $E$ be a set of representatives for the different equivalence classes of $\sim$. For each $e \in E$, let $R_{e}$ be a unital non-associative ring and put $R=\oplus_{e \in \mathrm{ob}(G)} R_{e}$. For each $g \in \operatorname{mor}(G)$, let $e(g)$ denote the unique element in $E$ such that $c(g) \sim e(g)$. For each $g \in \operatorname{mor}(G)$, put $R_{g}=R_{e(g)}$ and let $\alpha_{g}: R_{g^{-1}} \rightarrow R_{g}$ be the identity map. Then $\left\{\alpha_{g}: R_{g^{-1}} \rightarrow R_{g}\right\}_{g \in \operatorname{mor}(G)}$ is a partial action of the groupoid $G$ on $R$. The corresponding partial skew groupoid $\operatorname{ring} R \star_{\alpha} G$ coincides with the groupoid ring $R[G]$ of $G$ over $R$. Using the above notation, we get the following generalization of Connell's classical result for group rings [8, Theorem 1].

Theorem 4.1. The groupoid ring $R[G]$ is left (right) artinian if and only if $\operatorname{mor}(G)$ is finite and for each $e \in E$, the ring $R_{e}$ is left (right) artinian.

Proof. This follows immediately from Theorem 1.2 .

Remark 2. E. I. Zelmanov [38] has shown that if the semigroup ring $R[S]$ is left (or right) artinian for an associative ring $R$, then the semigroup $S$ must be finite. In [26] I. B. Kozhukhov showed that Zelmanov's result can not be generalized to (non-associative) magma rings. Theorem 4.1 appears to be the first generalization of Connell's result which applies to non-associative rings.

Let $I$ be a non-empty set and suppose that $T$ is a unital non-associative ring. Define a groupoid $\mathcal{I}$ in the following way. As objects of $\mathcal{I}$ we take the elements of $I$. As morphisms of $\mathcal{I}$ we take the elements of $I \times I$. Take $i, j, k \in I$. We put $d(i, j)=j$ and $c(i, j)=i$. The composition of $(i, j)$ with $(j, k)$, denoted $(i, j)(j, k)$, is defined to be $(i, k)$. The equivalence relation $\sim$, from above, only has one equivalence class for the groupoid $\mathcal{I}$. In other words, $\mathcal{I}$ is connected. So if we let $R_{e}=T$, for $e \in \mathrm{ob}(G)$, then the skew groupoid $\operatorname{ring} R \star_{\alpha} \mathcal{I}$ coincides with the generalized matrix ring $M_{I}(T)$. With the above notation, we get the following result. 
Corollary 4.2. The generalized matrix ring $M_{I}(T)$ is left (right) artinian if and only if $I$ is finite and $T$ is left (right) artinian.

Proof. This follows immediately from Theorem 4.1,

Given a field $K$ and a group $G$ one may define the partial group algebra $K_{\text {par }}[G]$ (see e.g. [11]).

Corollary 4.3. Let $K$ be a field. The partial group algebra $K_{\mathrm{par}}[G]$ is artinian if and only if $G$ is a finite group.

Proof. We first show the "if" statement. Using that $G$ is a finite group we get that the Brandt groupoid of $G$, denoted by $\Gamma$, is finite. By [11, Corollary 2.7], the partial group algebra $K_{\text {par }}[G]$ is isomorphic (as a $K$-algebra) to the groupoid algebra $K[\Gamma]$. The desired conclusion now follows immediately from Theorem 4.1 .

Now we show the "only if" statement. By [10, Theorem 6.9], $K_{\text {par }}[G]$ is isomorphic (as a $K$-algebra) to a certain partial skew group $\operatorname{ring} \mathcal{A} \rtimes_{\alpha^{\tilde{\pi}}} G$ associated to a partial action $\left\{\left\{\alpha_{g}^{\tilde{\pi}}\right\}_{g \in G},\left\{D_{g}\right\}_{g \in G}\right\}$. For each $g \in G, D_{g}$ contains the (non-zero) identity element $[g]\left[g^{-1}\right]$ (where $[g],\left[g^{-1}\right]$ are elements of Exel's semigroup). Hence, if $K_{\mathrm{par}}[G]$ is artinian, then, by Theorem $1.2, D_{g}=\{0\}$ for all but finitely many $g \in G$. This shows that $G$ is finite.

Remark 4.4. The "only if" statement of Corollary 4.3 can also be obtained through Zelmanov's theorem [38]. Indeed, $K_{\text {par }}[G]$ can be viewed as a semigroup algebra by Exel's semigroup.

\section{Applications to Leavitt path algebras}

A directed graph $E=\left(E^{0}, E^{1}, r, s\right)$ consists of two countable sets $E^{0}, E^{1}$ and maps $r, s: E^{1} \rightarrow E^{0}$. The elements of $E^{0}$ are called vertices and the elements of $E^{1}$ are called edges. If both $E^{0}$ and $E^{1}$ are finite sets, then we say that $E$ is finite. A vertex which emits no edge is called a sink. A vertex $v \in E^{0}$ such that $\left|s^{-1}(v)\right|=\infty$ is called an infinite emitter. A path $\mu$ in $E$ is a sequence of edges $\mu=\mu_{1} \ldots \mu_{n}$ such that $r\left(\mu_{i}\right)=s\left(\mu_{i+1}\right)$ for $i \in\{1, \ldots, n-1\}$. In such a case, $s(\mu):=s\left(\mu_{1}\right)$ is the source of $\mu, r(\mu):=r\left(\mu_{n}\right)$ is the range of $\mu$ and $n$ is the length of $\mu$. Recall that a path $\mu$ is called a cycle if $s(\mu)=r(\mu)$ and $s\left(\mu_{i}\right) \neq s\left(\mu_{j}\right)$ for every $i \neq j$. A graph $E$ without cycles is said to be acyclic.

Definition 5.1 (Leavitt path algebra [2]). Let $E$ be any directed graph and let $K$ be any field. The Leavitt path $K$-algebra $L_{K}(E)$ of $E$ with coefficients in $K$ is the $K$-algebra generated by a set $\left\{v \mid v \in E^{0}\right\}$ of pairwise orthogonal idempotents, together with a set of variables $\left\{f \mid f \in E^{1}\right\} \cup\left\{f^{*} \mid f \in E^{1}\right\}$, which satisfy the following relations:

(1) $s(f) f=f r(f)=f$, for all $f \in E^{1}$;

(2) $r(f) f^{*}=f^{*} s(f)=f^{*}$, for all $f \in E^{1}$;

(3) $f^{*} f^{\prime}=\delta_{f, f^{\prime}} r(f)$, for all $f, f^{\prime} \in E^{1}$;

(4) $v=\sum_{\left\{f \in E^{1} \mid s(f)=v\right\}} f f^{*}$, for every $v \in E^{0}$ for which $s^{-1}(v)$ is non-empty and finite.

In [20] D. Gonçalves and D. Royer have shown that the Leavitt path algebra $L_{K}(E)$ is isomorphic (as a $K$-algebra) to a partial skew group ring $D(X) \star_{\alpha} \mathbb{F}$, where $D(X)$ is a 
certain commutative $K$-algebra and $\mathbb{F}$ is the free group generated by $E^{1}$. For the benefit of the reader we shall briefly recall the construction of $D(X) \star_{\alpha} \mathbb{F}$.

Let $W$ denote the set of all finite paths in $E$, and let $W^{\infty}$ denote the set of all infinite paths in $E$. Let $\mathbb{F}$ denote the free group genereated by $E^{1}$. We are going to define a partial action of $\mathbb{F}$ on the set

$$
X=\{\xi \in W \mid r(\xi) \text { is a sink }\} \cup\left\{v \in E^{0} \mid v \text { is a sink }\right\} \cup W^{\infty} .
$$

For each $g \in \mathbb{F}$, let $X_{g}$ be defined as follows:

- $X_{e}:=X$, where $e$ is the identity element of $\mathbb{F}$.

- $X_{b^{-1}}:=\{\xi \in X \mid s(\xi)=r(b)\}$, for all $b \in W$.

- $X_{a}:=\left\{\xi \in X \mid \xi_{1} \xi_{2} \ldots \xi_{|a|}=a\right\}$, for all $a \in W$.

- $X_{a b^{-1}}:=\left\{\xi \in X \mid \xi_{1} \xi_{2} \ldots \xi_{|a|}=a\right\}=X_{a}$, for $a b^{-1} \in \mathbb{F}$ with $a, b \in W, r(a)=r(b)$ and $a b^{-1}$ in its reduced form.

- $X_{g}:=\emptyset$, for all other $g \in \mathbb{F}$.

Let $\theta_{e}: X_{e} \rightarrow X_{e}$ be the identity map. For $b \in W, \theta_{b}: X_{b^{-1}} \rightarrow X_{b}$ is defined by $\theta_{b}(\xi)=b \xi$ and $\theta_{b^{-1}}: X_{b} \rightarrow X_{b^{-1}}$ by $\theta_{b^{-1}}(\eta)=\eta_{|b|+1} \eta_{|b|+2} \ldots$ if $r(b)$ is not a sink, and by $\theta_{b^{-1}}(b)=r(b)$ if $r(b)$ is a sink. Finally, for $a, b \in W$ with $r(a)=r(b)$ and $a b^{-1}$ in reduced form, $\theta_{a b^{-1}}: X_{b a^{-1}} \rightarrow X_{a b^{-1}}$ is defined by $\theta_{a b^{-1}}(\xi)=a \xi_{(|b|+1)} \xi_{(|b|+2)} \ldots$, with inverse $\theta_{b a^{-1}}: X_{a b^{-1}} \rightarrow X_{b a^{-1}}$ defined by $\theta_{b a^{-1}}(\eta)=b \eta_{(|a|+1)} \eta_{(|a|+2)} \ldots$

One may easily verify that $\left\{\left\{X_{g}\right\}_{g \in \mathbb{F}},\left\{\theta_{g}\right\}_{g \in \mathbb{F}}\right\}$ is a partial action on the set level which induces a partial action on the algebra level. Indeed, we may define a partial action $\left\{\left\{F\left(X_{g}\right)\right\}_{g \in \mathbb{F}},\left\{\alpha_{g}\right\}_{g \in \mathbb{F}}\right\}$, where, for each $g \in \mathbb{F}, F\left(X_{g}\right)$ denotes the algebra of all functions from $X_{g}$ to $K$, and $\alpha_{g}: F\left(X_{g^{-1}}\right) \rightarrow F\left(X_{g}\right)$ by putting $\alpha_{g}(f)=f \circ \theta_{g^{-1}}$. Based on this partial action, we define another partial action in the following way:

For each $g \in \mathbb{F}$, and for each $v \in E^{0}$, define the characteristic maps $1_{g}:=\chi_{X_{g}}$ and $1_{v}:=\chi_{X_{v}}$, where $X_{v}=\{\xi \in X \mid s(\xi)=v\}$. Notice that $1_{g}$ is the identity element of $F\left(X_{g}\right)$. Finally, let

$$
D(X)=D_{e}=\operatorname{span}\left\{\left\{1_{g} \mid g \in \mathbb{F} \backslash\{0\}\right\} \cup\left\{1_{v} \mid v \in E^{0}\right\}\right\},
$$

(where span means the $K$-linear span) and, for each $g \in \mathbb{F} \backslash\{0\}$, let $D_{g} \subseteq F\left(X_{g}\right)$ be defined as $1_{g} D_{e}$, that is,

$$
D_{g}=\operatorname{span}\left\{1_{g} 1_{h} \mid h \in \mathbb{F}\right\} .
$$

By [20, Lemma 2.4], $D(X)$ is a $K$-algebra and $D_{g}$, for $g \in \mathbb{F}$, is an ideal of $D(X)$. Using that $\alpha_{g}\left(1_{g^{-1}} 1_{h}\right)=1_{g} 1_{g h}$ (see [20, Lemma 2.6]), for each $g \in \mathbb{F}$, it is clear that the restriction of $\alpha_{g}$ to $D_{g^{-1}}$ is a bijection onto $D_{g}$. By abuse of notation this restriction map will also be denoted by $\alpha_{g}$. Clearly, $\alpha_{g}: D_{g^{-1}} \rightarrow D_{g}$ is an isomorphism of $K$-algebras and, furthermore, $\left\{\left\{\alpha_{g}\right\}_{g \in \mathbb{F}},\left\{D_{g}\right\}_{g \in \mathbb{F}}\right\}$ is a partial action. By [20, Proposition 3.2] the map $\varphi: L_{K}(E) \rightarrow D(X) \star_{\alpha} \mathbb{F}$ defined by $\varphi(f)=1_{f} \delta_{f}, \varphi\left(f^{*}\right)=1_{f^{-1}} \delta_{f^{-1}}$, for all $f \in E^{1}$, and $\varphi(v)=1_{v} \delta_{e}$, for all $v \in E^{0}$, is an isomorphism of $K$-algebras.

Theorem 5.2. Let $K$ be a field and let $E$ be a directed graph. Consider $L_{K}(E)$, the Leavitt path $K$-algebra of $E$ with coefficients in $K$. The following five assertions are equivalent:

(i) $E$ is finite and acyclic; 
(ii) $L_{K}(E)$ is left artinian;

(iii) $L_{K}(E)$ is right artinian;

(iv) $L_{K}(E)$ is artinian;

(v) $L_{K}(E)$ is unital and semisimple.

Proof. Throughout this proof we are going to make use of the fact that the Leavitt path algebra $L_{K}(E)$ is isomorphic to the partial skew group ring $D(X) \star_{\alpha} \mathbb{F}$, as described above.

(ii) $\Rightarrow$ (iii): If we can show that $D(X)$ is left artinian and that $D_{g}=\{0\}$ for all but finitely many $g \in \mathbb{F}$, then it follows by Theorem 1.2 that $D(X) \star_{\alpha} \mathbb{F}$ is an artinian ring.

By the finiteness and acyclicity of $E$ we immediately conclude that there are no infinite paths in $E$ and that $W$, the set of all finite paths in $E$, is a finite set. Hence, using the notation of [20] we see that $X=\{\xi \in W \mid r(\xi)$ is a sink $\} \cup\left\{v \in E^{0} \mid v\right.$ is a sink $\}$ is a finite set. Using that $W$ is finite, we conclude that $X_{g}=\emptyset$ for all but finitely many $g \in \mathbb{F}$. Hence, $1_{g}=0$ for all but finitely many $g \in \mathbb{F}$. Using this, we immediately conclude that $D_{g}=\{0\}$ for all but finitely many $g \in \mathbb{F}$, and moreover, we notice that it turns $D(X)$ into a finite-dimensional $K$-vector space. Hence, $D(X)$ is artinian.

(iii) $\Rightarrow$ (ii): Suppose that $L_{K}(E) \cong D(X) \star_{\alpha} \mathbb{F}$ is left artinian. By Theorem 1.3, we get that $D_{g}=\{0\}$ for all but finitely many $g \in \mathbb{F}$.

We claim that there can be no infinite path in $E$, i.e. $W^{\infty}=\emptyset$. Clearly, every cycle gives rise to an infinite path. Thus, if we assume that the claim holds, then $E$ must be acyclic. Now we prove the claim. Seeking a contradiction, suppose that $E$ contains an infinite path $\xi=\xi_{1} \xi_{2} \xi_{3} \ldots$. By taking (finite) initial subpaths of $\xi$, which are elements of $W$ and hence also of $\mathbb{F}$, we can form an infinite chain of nested subsets

$$
X_{\xi_{1}} \supseteq X_{\xi_{1} \xi_{2}} \supseteq X_{\xi_{1} \xi_{2} \xi_{3}} \supseteq \cdots
$$

which are all non-empty since they contain $\xi$. Thus, the ideals

$$
D_{\xi_{1}}, \quad D_{\xi_{1} \xi_{2}}, \quad D_{\xi_{1} \xi_{2} \xi_{3}}, \quad \ldots
$$

are all non-zero. Hence, $D_{g} \neq\{0\}$ for infinitely many $g \in \mathbb{F}$. This is a contradiction.

Now we show that $E$ must be finite. Seeking a contradiction, suppose that $E^{0}=$ $\left\{v_{1}, v_{2}, v_{3}, \ldots\right\}$ is infinite. Notice that $L_{K}(E)=\oplus_{v \in E^{0}} L_{K}(E) v$ and consider the following descending chain of left ideals of $L_{K}(E)$.

$$
L_{K}(E) \supseteq \oplus_{v \in E^{0} \backslash\left\{v_{1}\right\}} L_{K}(E) v \supseteq \oplus_{v \in E^{0} \backslash\left\{v_{1}, v_{2}\right\}} L_{K}(E) v \supseteq \oplus_{v \in E^{0} \backslash\left\{v_{1}, v_{2}, v_{3}\right\}} L_{K}(E) v \supseteq \ldots
$$

It is easy to see that the above chain never stabilizes, using the fact that every pair of vertices in $E^{0}$ are orthogonal idempotents. Hence, $L_{K}(E)$ is not left artinian. This is a contradiction. We conclude that $E^{0}$ is finite.

We now proceed to show that $E^{1}$ must be finite. Using that $E^{0}$ is finite, it is sufficient to show that $E^{0}$ contains no infinite emitter. Seeking a contradiction, suppose that there is a vertex $v \in E^{0}$ which is an infinite emitter. Since $E^{0}$ is finite, there must exist some $u \in E^{0}$ such that the set $I=\left\{e \in E^{1} \mid s(e)=v\right.$ and $\left.r(e)=u\right\}$ is infinite.

Case 1: $(u$ is a sink)

Take $e \in I \subseteq W$ and consider the set $X_{e^{-1}}=\{\xi \in X \mid s(\xi)=u\}$ which is non-empty since it contains $u$. Hence, $D_{e^{-1}}$ is non-zero for (infinitely many) $e \in I$. This is a contradiction. 
Case 2: $(u$ is not a sink)

We have already shown that $E$ contains no infinite path, i.e. $W^{\infty}=\emptyset$. Hence, there must exist at least one path from $u$ to a sink $w$. Let us call it $\eta$. Take $e \in I$ and consider the set $X_{e^{-1}}=\{\xi \in X \mid s(\xi)=u\}$ which is non-empty since it contains $\eta$. Hence, $D_{e^{-1}}$ is non-zero for (infinitely many) $e \in I$. This is a contradiction.

This shows that $E^{1}$ (and hence also $E$ ) is finite.

(iii) $\Leftrightarrow($ iii) $\Leftrightarrow($ iv) : This follows immediately from Theorem 1.3, using the fact that $D(X)$ is commutative.

$(\mathrm{iv}) \Rightarrow(\mathbb{\nabla})$ : By [2, Proposition 6.3] we know that $L_{K}(E)$ is semiprimitive, i.e. $J\left(L_{K}(E)\right)=$ $\{0\}$. Hence, if $L_{K}(E)$ is left artinian it must also be semisimple (see e.g. [28, Theorem 4.14]). Using that (iv) $\Leftrightarrow$ (ii) we get that $E$ is finite and hence, in particular, $L_{K}(E)$ is unital. $(\mathrm{v}) \Rightarrow(\mathrm{iv})$ : This is clear.

Remark 5.3. The essence of the above result has previously been shown in [3] using a different technique.

Recall that a subset $H \subseteq E^{0}$ is said to be hereditary if for any $e \in E^{1}$ we have that $s(e) \in H$ implies $r(e) \in H$. A hereditary subset $H \subseteq E^{0}$ is called saturated if whenever $0<\# s^{-1}(v)<\infty$, then $\left\{r(e) \in H \mid e \in E^{1}\right.$ and $\left.s(e)=v\right\} \subseteq H$ implies $v \in H$.

Corollary 5.4. If $E$ is a finite and acyclic graph such that the only hereditary and saturated subsets of $E^{0}$ are $\emptyset$ and $E^{0}$, then the Leavitt path algebra $L_{K}(E)$ is a simple unital artinian ring.

Proof. Since $E$ is acyclic it trivially satisfies condition (L), see [19]. By [19, Theorem 3.5] we conclude that $L_{K}(E)$ is simple. We get from Theorem 5.2 that $L_{K}(E)$ is a simple unital artinian ring.

\section{Morita equivalence, Globalization, Noetherianity and Maschke-Type RESULTS}

In this section, we use globalization and Morita equivalence to deduce necessary and sufficient conditions for the artinianity, noetherianity and two Maschke-type reults. We adapt the approach taken in [17] to the groupoid situation. To this end, we recall the following.

Definition 6.1. Following [5], we say that a global action $\beta=\left\{\beta_{g}: T_{g^{-1}} \rightarrow T_{g}\right\}_{g \in \operatorname{mor}(G)}$ of a groupoid $G$ on an associative ring $T$ is a globalization of a partial action $\alpha=\left\{\alpha_{g}\right.$ : $\left.R_{g^{-1}} \rightarrow R_{g}\right\}_{g \in \operatorname{mor}(G)}$ of $G$ on an associative $\operatorname{ring} R$ if, for each $e \in \mathrm{ob}(G)$, there exists a ring monomorphism $\psi_{e}: R_{e} \rightarrow T_{e}$ such that, for every $g \in \operatorname{mor}(G)$ and every $a \in R_{g^{-1}}$, the following hold:

(i) $\psi_{e}\left(R_{e}\right) \unlhd T_{e}$, i.e. $\psi_{e}\left(R_{e}\right)$ is a two-sided ideal of $T_{e}$;

(ii) $\psi_{c(g)}\left(R_{g}\right)=\psi_{c(g)}\left(R_{c(g)}\right) \cap \beta_{g}\left(\psi_{d(g)}\left(R_{d(g)}\right)\right)$;

(iii) $\beta_{g}\left(\psi_{d(g)}(a)\right)=\psi_{c(g)}\left(\alpha_{g}(a)\right)$;

(iv) and $T_{g}=\sum_{c(h)=c(g)} \beta_{h}\left(\psi_{d(h)}\left(R_{d(h)}\right)\right)$. 
Remark 6.2. Throughout this section, let $\alpha=\left\{\alpha_{g}: R_{g^{-1}} \rightarrow R_{g}\right\}_{g \in \operatorname{mor}(G)}$ be a unital partial action of groupoid $G$ on a ring $R$. It follows from [5, Theorem 2.1] that $\alpha$ admits a globalization $\beta=\left\{\beta_{g}: T_{g^{-1}} \rightarrow T_{g}\right\}_{g \in \operatorname{mor}(G)}$. From [5, Remark 2.3], we may, for the rest of the section, fix $\alpha$ and $\beta$ so that $\psi_{e}=\mathrm{id}_{R_{e}}$. Then, $R_{e} \unlhd T_{e}$ and $T_{e} 1_{R_{e}}=R_{e}$, for any $e \in \mathrm{ob}(G)$. We shall also assume that $T=\bigoplus_{e \in \mathrm{ob}(G)} T_{e}$.

Definition 6.3. We say that $\alpha$ is of finite type, if for any $e \in \mathrm{ob}(G)$ there are $g_{1}, \ldots, g_{n} \in$ $G(-, e)$ such that $R_{c(g)}=\sum_{i=1}^{n} R_{g g_{i}}$, for any $g \in G(e,-)$, where $G(-, e)=\{g \in G \mid c(g)=$ $e\}$ and $G(e,-)=\{g \in G \mid d(g)=e\}$.

Proposition 6.4. The following three assertions are equivalent:

(i) $\alpha$ is of finite type;

(ii) For any $e \in \mathrm{ob}(G)$, there exists $g_{1}, \ldots, g_{n} \in G(-, e)$ such that $T_{e}=\sum_{i=1}^{n} \beta_{g_{i}}\left(R_{d\left(g_{i}\right)}\right)$;

(iii) For any $e \in \operatorname{ob}(G)$, the ring $T_{e}$ is unital.

Proof. (ii) $\Rightarrow$ (iii): Let $e \in \mathrm{ob}(G)$. Then by (iv) in the definition of a globalization one has that $T_{e}=\sum_{c(h)=e} \beta_{h}\left(R_{d(h)}\right)$. Since $\alpha$ is of finite type there are $g_{1}, \ldots, g_{n} \in G(-, e)$ such that $R_{d(h)}=\sum_{i=1}^{n} R_{h^{-1} g_{i}}$, for each $h \in G$ with $c(h)=e$. Then $\beta_{h}\left(R_{d(h)}\right)=\sum_{i=1}^{n} \beta_{h}\left(R_{h^{-1} g_{i}}\right)$. From this we get that

$$
\begin{aligned}
\sum_{i=1}^{n} \beta_{g_{i}}\left(R_{d\left(g_{i}\right)}\right) \subseteq T_{e}=\sum_{c(h)=e} \beta_{h}\left(R_{d(h)}\right)=\sum_{c(h)=e} \sum_{i=1}^{n} \beta_{h}\left(R_{h^{-1} g_{i}}\right) & =\sum_{i=1}^{n} \sum_{c(h)=e} \beta_{h}\left(R_{h^{-1} g_{i}}\right) \\
& \subseteq \sum_{i=1}^{n} \beta_{g_{i}}\left(R_{d\left(g_{i}\right)}\right) .
\end{aligned}
$$

using that $\beta_{h}\left(R_{h^{-1} g_{i}}\right)=\beta_{h}\left(R_{c\left(h^{-1} g_{i}\right)}\right) \cap \beta_{h} \beta_{h^{-1} g_{i}}\left(R_{d\left(h^{-1} g_{i}\right)}\right) \subseteq \beta_{g_{i}}\left(R_{d\left(g_{i}\right)}\right)$, for each $h \in$ $G(-, e)$. Since $\sum_{i=1}^{n} \beta_{g_{i}}\left(R_{d\left(g_{i}\right)}\right) \subseteq T_{e}$ we conclude that $T_{e}=\sum_{i=1}^{n} \beta_{g_{i}}\left(R_{d\left(g_{i}\right)}\right)$, as desired.

(iii) $\Rightarrow$ (iii) This follows from the fact that $T_{e}$ is a finite sum of unital rings (see [10, Lemma $4.4])$.

(iii) $\Rightarrow$ (ii) There are $h_{1}, \ldots, h_{n} \in G(-, e)$ such that $1_{T_{e}}=\sum_{i=1}^{n} \beta_{h_{i}}\left(r_{i}\right)$, where $r_{i} \in R_{d\left(h_{i}\right)}$, for $i \in\{1, \ldots, n\}$. Thus, for any $g \in G(e,-)$ we get that $1_{T_{c(g)}}=\beta_{g}\left(1_{T_{e}}\right)=\sum_{i=1}^{n} \beta_{g h_{i}}\left(r_{i}\right)$. Since $R_{c(g)} \unlhd T_{c(g)}$ one has that $1_{R_{c(g)}}=\sum_{i=1}^{n} \beta_{g h_{i}}\left(r_{i}\right) 1_{R_{c(g)}} \in \sum_{i=1}^{n}\left[\beta_{g h_{i}}\left(R_{d\left(g h_{i}\right)}\right) \cap R_{c\left(g h_{i}\right)}\right]=$ $\sum_{i=1}^{n} \beta_{g h_{i}}\left(R_{g h_{i}}\right)$ which is an ideal of $R_{c(g)}$. Hence $R_{c(g)}=\sum_{i=1}^{n} \beta_{g h_{i}}\left(R_{g h_{i}}\right)$ and $\alpha$ is of finite type.

Remark 6.5. The partial skew groupoid $\operatorname{ring} R \star_{\alpha} G$ is, in the sense of [29], a groupoid graded ring. Therefore, from [29, Proposition 2.1.1] (or in a more general context [30, Proposition 5]), it follows that $R \star_{\alpha} G$ is unital if $\mathrm{ob}(G)$ is finite. In that case, if $\alpha$ is of 
finite type, it follows from Proposition 6.4 and [5, Theorem 3.2], that $R \star_{\alpha} G$ and $T \star_{\beta} G$ are Morita equivalent.

Corollary 6.6. If ob $(G)$ is finite, then $T$ is right/left noetherian (artinian) if and only $R$ is right/left noetherian (artinian) and $\alpha$ is of finite type.

Proof. Suppose that $\mathrm{ob}(G)$ is finite. First we show the "if" part. Suppose that $R$ is right/left noetherian (artinian) and $\alpha$ is of finite type. Then by Proposition 6.4(ii), each $T_{e}$ is a finite sum of right/left noetherian (artinian) $T_{e}$-modules, which implies that $T_{e}$ is right/left noetherian (artinian), for all ob $(G)$. Since $T=\bigoplus_{e \in \mathrm{ob}(G)} T_{e}$, it follows from Proposition 2.5, that $T=\bigoplus_{e \in \mathrm{ob}(G)} T_{e}$ is right/left noetherian (artinian). Now we show the "only if" part. If $T$ is right/left noetherian (artinian), so is $R$, because $R$ is an ideal of $T$. It remains to show that $\alpha$ is of finite type. Seeking a contradiction, suppose that there is $e \in \mathrm{ob}(G)$ such that $T_{e}$ is not a finite sum of ideals $\beta_{g}\left(R_{d(g)}\right)$ with $g \in G(-, e)$. Then there is an infinite ascending sequence of these sums in which any term is generated by a central idempotent of $T_{e}$. This contradicts the fact that $T_{e}$ is noetherian. Also, the annihilators of these idempotents give an infinite descending sequence of ideals of $T_{e}$, which contradicts the fact that $T_{e}$ is right artinian.

Corollary 6.7. If $\mathrm{ob}(G)$ is finite, then $R \star_{\alpha} G$ is left/right artinian if and only if $T \star_{\beta} G$ is left/right artinian and $\alpha$ is of finite type. In that case, both $R$ and $T$ are left/right artinian.

Proof. Suppose that $R \star_{\alpha} G$ is left/right artinian. By Proposition 3.4, we get that $R_{g}=\{0\}$, for all but finitely many $g \in \operatorname{mor}(G)$. Thus, from (iv) in the definition of a globalization, we get that each $T_{g}$ is a finite sum of unital rings. From Proposition 6.4(iii), we thus get that $\alpha$ is of finite type. Thus from Morita equivalence it follows that $T \star_{\beta} G$ is left/right artinian. Conversely, if $T \star_{\beta} G$ is left/right artinian and $\alpha$ is of finite type, then again by Morita equivalence, we get that $R \star_{\alpha} G$ is left/right artinian. The last part follows from Proposition 3.4 .

Corollary 6.8. If ob $(G)$ is finite and $\alpha$ is of finite type, then $R \star_{\alpha} G$ is left/right noetherian if and only if $T \star_{\beta} G$ is left/right noetherian. In that case, both $R$ and $T$ are left/right noetherian.

Proof. This follows from Morita equivalence and Proposition 3.4 .

We shall now prove some Maschke-type results for partial skew groupoid rings associated with unital partial actions of groupoids on rings (see Theorem 6.11 and Corollary 6.14).

Lemma 6.9. If $\mathrm{ob}(G)$ is finite, then $T$ is semisimple if and only if $R$ is semisimple and $\alpha$ is of finite type.

Proof. Suppose that $T$ is semisimple. Then $T$ has an identity. Thus, from Proposition 6.4, it follows that $\alpha$ is of finite type. Also, since $R$ is an ideal of $T$, it follows that $R$ is semisimple. Conversely, suppose that $R$ is semisimple and that $\alpha$ is of finite type. Then each $T_{e}$, for $e \in \mathrm{ob}(G)$, is a finite sum of semisimple rings. Thus, since $\mathrm{ob}(G)$ is finite, $T$ is semisimple. 
Lemma 6.10. For any positive integer $m$, the following hold:

(a) $R$ has $m$-additive torsion if and only if $T$ has $m$-additive torsion;

(b) if $T$ is unital, then $m$ is invertible in $R$ if and only if $m$ is invertible in $T$.

Proof. Analogous to the proof of [17, Proposition 1.22].

Theorem 6.11. If $\operatorname{mor}(G)$ is finite, $R$ is semisimple and for every $e \in \operatorname{ob}(G),\left|G_{e}\right|$ is invertible in $R$, then $R \star_{\alpha} G$ is semisimple.

Proof. From Lemma 6.9 it follows that $T$ is semisimple. From Lemma 6.10(b), we get that for every $e \in \operatorname{ob}(G),\left|G_{e}\right|$ is invertible in $T$. Thus, since $T \star_{\beta} G$ is strongly graded by $G$, we get, from [30, Proposition 10(b)], that $T \star_{\beta} G$ is semisimple. From Morita equivalence, we get that $R \star_{\alpha} G$ is semisimple.

Definition 6.12. Suppose that $\operatorname{mor}(G)$ is finite. The trace map was defined in [5] as

$$
\operatorname{tr}_{\alpha}: R \ni x \mapsto \sum_{g \in \operatorname{mor}(G)} \alpha_{g}\left(x 1_{g^{-1}}\right) \in R^{\alpha},
$$

where $R^{\alpha}=\left\{x \in R \mid \alpha_{g}\left(x 1_{g^{-1}}\right)=x 1_{g}\right.$, for all $\left.g \in G\right\}$.

Theorem 6.13. Suppose that $\operatorname{mor}(G)$ is finite. Let $V$ be a left $R \star_{\alpha} G$-module and let $W$ be a submodule of $V$. If $\operatorname{tr}_{\alpha}\left(1_{R}\right)$ is invertible in $R$ and $W$ is a direct summand of $V$ as an $R$-module, then $W$ is a direct summand of $V$ as an $R \star_{\alpha} G$-module.

Proof. We will only provide a sketch of the full proof as it is analogous to the proof of [17, Theorem 3.2]. Suppose that $\pi: V \rightarrow W$ is an $R$-projection. Put $l=\left(\operatorname{tr}_{\alpha}\left(1_{R}\right)\right)^{-1}$. For any $v \in V$, we define $\psi(v)=l \sum_{g \in \operatorname{mor}(G)} 1_{g^{-1}} \delta_{g^{-1}} \pi\left(1_{g} \delta_{g} v\right)$. Clearly, this yields a well-defined map $\psi: V \rightarrow W$ and one can check that $\psi$ is in fact a left $R \star_{\alpha} G$-module homomorphism. We notice that $\psi(w)=w$ for any $w \in W$. Thus, $\psi$ is a projection onto $W$.

The following is an immediate consequence of Theorem 6.13.

Corollary 6.14. If $\operatorname{mor}(G)$ is finite, $R$ is semisimple and $\operatorname{tr}_{\alpha}\left(1_{R}\right)$ is invertible in $R$, then $R \star_{\alpha} G$ is semisimple.

\section{REFERENCES}

[1] F. Abadie, On partial actions and groupoids, Proc. Amer. Math. Soc., 132 (2004), no. 4, $1037-1047$.

[2] G. Abrams and G. Aranda Pino, The Leavitt path algebras of arbitrary graphs, Houston J. Math. 34 (2008), no. 2, 423-442.

[3] G. Abrams, G. Aranda Pino and M. Siles Molina, Finite-dimensional Leavitt path algebras, J. Pure Appl. Algebra 209 (2007), no. 3, 753-762.

[4] D. Bagio, D. Flôres and A. Paques, Partial actions of ordered groupoids on rings. J. Algebra Appl. 9 (2010), no. 3, 501-517.

[5] D. Bagio and A. Paques, Partial groupoid actions: globalization, Morita theory, and Galois theory, Comm. Algebra 40 (2012), no. 10, 3658-3678.

[6] D. Bagio and H. Pinedo, On the separability of the partial skew groupoid ring. preprint.

[7] G. Boava and R. Exel, Partial crossed product description of the $\mathrm{C}^{*}$-algebras associated with integral domains, Proc. Amer. Math. Soc. 141 (2013), no. 7, 2439-2451. 
[8] I. G. Connell, On the group ring, Canad. J. Math. 15 (1963), 650-685.

[9] M. Dokuchaev, Partial actions: a survey. Group, algebras and applications, Contemp. Math. 537 (2011), 173-184.

[10] M. Dokuchaev and R. Exel, Associativity of crossed products by partial actions, enveloping actions and partial representations, Trans. Amer. Math. Soc. 357 (2005), no. 5, 1931-1952.

[11] M. Dokuchaev, R. Exel and P. Piccione, Partial representations and partial group algebras, J. Algebra 226 (2000), no. 1, 505-532.

[12] A. Elduque and H. C. Myung, Mutations of alternative algebras. Mathematics and its applications, 278. Kluwer Academic Publishers Group, Dordrecht, 1994.

[13] R. Exel, Circle actions on $\mathrm{C}^{*}$-algebras, partial automorphisms, and a generalized Pimsner-Voiculescu exact sequence, J. Funct. Anal. 122 (1994), no. 2, 361-401.

[14] R. Exel, The Bunce-Deddens algebras as crossed products by partial automorphisms, Bol. Soc. Brasil. Mat. (N.S.) 25 (1994), no. 2, 173-179.

[15] R. Exel, Approximately finite C*-algebras and partial automorphisms, Math. Scand. 77 (1995), no. 2, 281-288.

[16] R. Exel and M. Laca, Cuntz-Krieger algebras for infinite matrices, J. Reine Angew. Math. 512 (1999), 119-172.

[17] M. Ferrero and J. Lazzarin, Partial actions and partial skew group rings, J. Algebra 319 (2008), no. $12,5247-5264$.

[18] N. D. Gilbert, Actions and expansions of ordered groupoids, J. Pure Appl. Algebra 198 (2005), no. $1-3,175-195$.

[19] D. Gonçalves, J. Öinert and D. Royer, Simplicity of partial skew group rings with applications to Leavitt path algebras and topological dynamics, J. Algebra 420 (2014), 201-216.

[20] D. Gonçalves and D. Royer, Leavitt path algebras as partial skew group rings, Comm. Algebra 42 (2014), no. 8, 3578-3592.

[21] D. Gonçalves and G. Yoneda, Free path groupoid grading on Leavitt path algebras, Internat. J. Algebra Comput. 26 (2016), no. 6, 1217-1235.

[22] V. Gould and C. Hollings, Partial actions of inverse and weakly left E-ample semigroups, J. Aust. Math. Soc. 86 (2009), no. 3, 355-377.

[23] V. Gould and C. Hollings, Actions and partial actions of inductive constellations, Semigroup Forum 82 (2011), no. 1, 35-60.

[24] C. Hollings, Partial actions of monoids, Semigroup Forum 75 (2007), no. 2, 293-316.

[25] J. Kellendonk and M. V. Lawson, Partial actions of groups, Internat. J. Algebra Comput. 14 (2004), no. $1,87-114$.

[26] I. B. Kozhukhov, Artinian groupoid rings, Semigroup Forum 56 (1998), no. 3, 437-441.

[27] G. Kudryavtseva, Partial monoid actions and a class of restriction semigroups, J. Algebra. 429 (2015), 342-370.

[28] T. Y. Lam, A first course in noncommutative rings. Second edition. Graduate Texts in Mathematics, 131. Springer-Verlag, New York, 2001.

[29] P. Lundström, The category of groupoid graded modules, Colloquium Mathematicum 100 (2004), no. 2, 195-211.

[30] P. Lundström, Separable Groupoid Rings, Comm. Algebra 34 (2006), no. 8, 3029-3041.

[31] P. Lundström and J. Öinert, Commutativity and ideals in category crossed products, Proc. Est. Acad. Sci. 59 (2010), no. 4, 338-346.

[32] D. B. McAlister and N. R. Reilly, E-unitary covers for inverse semigroups, Pacific J. Math. 68 (1977), no. $1,161-174$.

[33] M. Megrelishvili and L. Schröder, Globalization of confluent partial actions on topological and metric spaces, Topology Appl. 145 (2004), nos. 1-3, 119-145. 
[34] P. Nystedt, J. Öinert and J. Richter, Non-associative Ore Extensions, arXiv:1509.01436 [math.RA], preprint.

[35] J. K. Park, Artinian skew group rings, Proc. Amer. Math. Soc 75 (1979), no. 1, 1-7.

[36] D. S. Passman, Radical of twisted group rings. II, Proc. London Math. Soc. (3) 22 (1971), 633-651.

[37] L. H. Rowen, Ring Theory. Vol. I. Pure and Applied Mathematics, 127. Academic Press, Inc., Boston, MA, 1988.

[38] E. I. Zelmanov, Semigroup algebras with identities, Sibirsk. Mat. Z. 18 (1977), no. 4, 787-798.

[39] K. A. Zhevlakov, A. M. Slinko, I. P. Shestakov and A. I. Shirshov, Rings that are nearly associative, Academic Press, New York (1982). 